\title{
NAISSANCE DE LA BIOLOGIE ET REDISTRIBUTION DES SAVOIRS
}

\author{
Roselyne REY
}

REsumE : Le champ de l'histoire naturelle, longtemps descriptif et classificatoire, connaît tout au long du xvif siècle, un ensemble de transformations dues au développement croissant d'un point de vue physiologique sur les êtres vivants. Si, dans un premier temps, les disciplines traditionnelles (botanique, zoologie) voient des aspects particuliers se développer en leur sein (physique végétale, anatomie et physiologie comparées), la question se pose peu à peu de l'émergence d'une science du vivant, dépassant le clivage traditionnel des trois règnes, consciente de l'unité que représente le monde vivant par opposition au monde inorganique. L'apparition presque simultanée en Europe du terme biologie (Roze, Treviranus, Lamarck) ou de son substitut zoonomie (E. Darwin) ne constitue cependant qu'une naissance avortée, qui perturbe les classifications antérieures, sans aboutir immédiatement à la reconnaissance de la biologie comme discipline. L'objet dè cet article est d'analyser les conditions conceptuelles qui permettent cette naissance, d'examiner les processus de redistribution des savoirs qu'elle entraîne et d'étudier les redéfinitions et les redécoupages qui s'opèrent dans les catégories utilisées dans les institutions académiques, les périodiques ou les dictionnaires.

La formule "naissance de la biologie " recèle suffisamment d'équivoques pour qu'il soit nécessaire d'apporter quelques précisions liminaires : ne parle-t-on pas, en effet, de la " biologie " d'Aristote, et cela ne suppose-t-il pas une sorte de continuité, de permanence de la discipline, définie par son objet d'étude, rendant dès lors nul et non avenu un quelconque acte de naissance de la biologie dans la seconde moitié du xvir siècle? Il faut cependant rappeler sans ambiguité que la façon dont Aristote pose les problèmes théoriques du vivant et le cadre conceptuel dans lequel ils se formulent à l'aube du $\mathrm{xxx}^{e}$ siècle ont quelque chose d'incommensurable :

« il faut fermement maintenir que cette biologie [celle d'Aristote] ne peut être pour nous, seulement inachevée, mais qu'elle nous est radicalement 
étrange, en ce que, produite dans un univers révolu, elle tente de répondre à des questions que nous ne nous posons plus " ${ }^{1}$.

D'un autre côté, à parler de biologie pour cette période comprise entre 1750 et 1830 , ne risque-t-on pas de plaquer une catégorie de la classification qui ne prendra son sens que plus tard, à partir de la formulation qu'en a donnée Auguste Comte dans le Cours de philosophie positive ${ }^{2}$, et, au-delà, avec la création de la Société de biologie en $1848^{3}$ ? Dans les classifications utilisées dans le cours du xvir ${ }^{\mathrm{e}}$ siècle - Encyclopédie, Encyclopédie méthodique, pratique implicite de la division des sciences à travers la désignation des sections de l'Académie des sciences ou des rubriques des périodiques -, non seulement le terme de biologie n'apparaît pas, mais l'idée même de regrouper sous un vocable unique, quel qu'il soit, la science du vivant, ne paraît pas s'imposer. Bien que le contexte philosophique fourni par la philosophie de Condillac impose une vigilance et une rigueur particulières dans l'usage des signes linguistiques, il ne nous paraît pas possible de limiter la réflexion à la relation entre le mot, ou ses possibles substituts, et la chose : certes, l'absence d'un terme pour désigner une science est toujours signifiante, mais elle ne dit pas forcément l'absence des objets d'étude qui constitueront cette science; et inversement, l'apparition du terme, de manière presque conjointe en Allemagne et en France, chez Roze, Treviranus et Lamarck constitue un indice de changements, plutôt qu'une preuve définitive de l'avènement d'une nouvelle division des sciences. La première occurrence du vocable biologie en français, qui se trouve comme l'on sait dans l'Hydrogéologie de Lamarck, peut, à la réflexion, apparaître singulièrement ambiguë, situant la biologie comme partie subordonnée à « la physique terrestre, c'est-à-dire tout ce qu'on observe à la surface et dans la croûte externe du globe "; Lamarck ajoute :

« Enfin les observations que j'ai faites sur les corps vivants et dont j'ai exposé les principaux résultats dans le Discours d'ouverture de mon cours de

1. Pierre Pellegrin, La Classification des animaux chez Aristote. Statut de la biologie et unité de l'aristotélisme, Paris, Les Belles Lettres, 1982, p. 10.

2. Auguste Comte, Cours de philosophie positive, présentation et notes par Michel SerRes, François Dagognet, Allal Sinaceur, Paris, Hermann, 1975 ; dans la $40^{c}$ leçon, p. 685, Comte indique : « si l'idée de vie est réellement inséparable de celle d'organisation, l'une et l'autre ne sauraient s'isoler davantage [...] de celle d'un milieu spécial en relation déterminée avec elles. Il en résulte donc un troisième aspect élémentaire, non moins indispensable, du sujet fondamental de la biologie, savoir la théorie générale des milieux organiques, et de leur action sur l'organisme, envisagée d'une manière abstraite. " Pour une réflexion d'ensemble, voir Claire SALOMON-BAYET, L'Institution de la science et l'expérience du vivant, Paris, Flammarion, 1978.

3. La création de la Société de biologie est étroitement liée au courant positiviste, comme le montre la liste des premiers membres ainsi que les orientations exprimées par Charles Robin dans son Discours d'ouverture. 
l'an 9 au Muséum, feront le sujet de ma biologie, troisième et dernière partie de la Physique terrestre. Il existe un grand nombre d'objets qui attestent que l'organisation des corps vivants, c'est-à-dire la conformation interne de ces corps et de leurs parties est uniquement le résultat des fluides qu'ils contiennent $"{ }^{4}$.

Cette définition, centrée sur le mouvement des fluides, ne fait guère de place à la spécificité du vivant. Loin donc de proposer une lecture univoque et immédiate des liens entre la désignation d'une science par un nom particulier, nouveau, et son " existence ", une étude sur l'introduction de la biologie dans la classification des sciences doit nécessairement s'interroger sur les conditions de construction d'une nouvelle catégorie. Dans le processus qui conduit à cette émergence, il y a toujours déplacement des catégories préexistantes, bouleversement, voire subversion : la biologie en tant que science générale du vivant occupe une place qui était partiellement tenue par d'autres sciences, elle redécoupe des frontières, désorganise les clivages les mieux installés. Quelles sont les raisons qui remettent en cause la traditionnelle distinction entre les règnes, qui permettait de s'occuper, séparément, de minéralogie, de botanique et de zoologie?

Dans la seconde leçon du Cours de philosophie positive, Comte remarquait que la " physiologie ", définie comme "connaissance des lois générales de la vie ", supposait « la considération simultanée de la série organique, sans distinction de végétaux et d'animaux, distinction qui d'ailleurs s'efface de jour en jour à mesure que les phénomènes sont étudiés d'une manière approfondie $"{ }^{5}$. Ainsi la "physiologie " était placée en situation de concurrence avec la biologie : sous l'expression de "physiologie générale », Claude Bernard persista d'ailleurs à désigner "la connaissance et l'explication de toutes les manifestations de la vie ${ }^{6}$, cherchant à distinguer cette science en train de conquérir son indépendance d'une biologie comtienne au sens trop large, puisqu'elle se définissait en fonction du double point de vue statique et dynamique. Pourquoi substituer une autre appellation à celles, déjà englobantes, d'histoire naturelle, puis de sciences naturelles? Dans le cas qui nous occupe, le problème n'est pas tant l'apparition d'un nouvel objet, l'étude du vivant, que la reconnaissance de la spécificité de cet objet : pour qu'une science générale du vivant apparaisse, la délimitation entre vivant et non vivant doit l'emporter sur toutes les autres formes de représentation de la

4. Jean-Baptiste LAmARCK, Hydrogéologie, Paris, L'auteur, an X (1802), Appendice, p. 188.

5. A. СомтE, op. cit. supra n. $2,2^{\mathrm{e}}$ leçon, p. 57.

6. Claude BERnARD, Rapport sur les progrès et la marche de la physiologie générale en France, Paris, Imprimerie impériale, 1867, p. 2. 
nature. Pour simple qu'elle paraisse d'abord, cette « division générale de la nature, proclamée par Buffon ${ }^{7}$, n'a pourtant rien d'évident, elle n'a même que les apparences de la naturalité : l'acte qui a consisté à mettre au premier plan cette distinction est un produit historique, qui n'a été possible qu'en éliminant une série d'autres possibilités, qui ont pu être considérées comme tout aussi légitimes. Pourquoi ne pas isoler l'homme dans la nature, ne pas affirmer la continuité de la chaîne des êtres du minéral à l'animal, ne pas situer la ligne de démarcation entre l'organisé et l'inorganisé ? Selon quels critères opérer cette séparation, qui conduit aussi à définir les frontières du vivant? Tâche difficile entre toutes puisqu'à cette même époque charnière de la fin du xviI' siècle, on étudie les lois de l'organisation du cristal, sa capacité à reformer des figures géométriques régulières après perturbation...

La définition de la vie demeure problématique, d'autant plus que la question même de sa nature a pu être considérée comme une question métaphysique; les fondements de la biologie sont peut-être moins liés à la délimitation de l'objet de la science qu'à un point de vue sur ces objets, une méthode pour les étudier, l'institution enfin de concepts opératoires pour les cerner, et la mise au jour de systèmes de relations entre eux : liens par exemple entre structure et fonction, qui renvoient aussi aux rapports entre anatomie et physiologie, liens entre forme et fonction, entre organisme et milieu. Mais comment, par-delà l'infinie diversité des formes vivantes retrouver la généralité des lois de fonctionnement et des rapports ? Comment retrouver les éléments communs aux animaux et aux végétaux? Précisément par la recherche d'un niveau élémentaire commun, que l'Analyse, devenue la méthode heuristique par excellence ${ }^{8}$, a stimulée, sous la poussée conjuguée des Idéologues et des résultats apportés par la chimie lavoisienne et par les travaux mathématiques : niveau le plus simple de l'organisation ou de l'organique ${ }^{9}$, qui a été cherché tant

7. Georges Louis LeClerC, comte de Buffon a formulé nettement l'importance de cette division in Histoire naturelle, générale et particulière, Paris, Imprimerie royale, 1749, t. II, p. 2 : « Il me parâit que la division générale qu'on devrait faire de la matière est matière vivante et matière morte, au lieu de dire matière organisée et matière brute : le brut n'est que le mort "; sur ce point, voir Roselyne REY, "Buffon et le vitalisme ", in Buffon 88, Actes du Colloque international, Paris-Montbard-Dijon, textes réunis par J.-C. BEAUNE, S. BENoît, J. Gayon, J. Roger, D. Woronoff, Paris, Vrin, 1992, p. 399-413. Voir Jacques Roger, Les Sciences de la vie dans la pensée française du xvilf siècle, Paris, Colin, 1971, et Giulio BarSANT, « La Naissance de la biologie », in Histoire, nature et société. Mélanges en l'honneur de Jacques Roger, Paris, Klincksieck, 1994.

8. Sur les différents sens et le champ d'application de l'analyse, voir Éric Brian, « La foi du géomètre, métier et vocation de savant pour Condorcet vers 1770 ", Revue de synthèse, IV S., I, janv.-mars 1988, p. 46-53.

9. «Organique » n'a d'abord désigné que ce qui avait rapport aux organes (voir Encyclopédie ou Dictionnaire raisonné des sciences, des arts et des métiers, par M. DIDEROT et M. D'Alembert, Paris, $1751-1765$ (cité par la suite par Enc.), t. XI, 1765, p. 629 b) puis fut confondu avec « organisé " (voir, par ex., Pierre-Hubert Nysten, Dictionnaire de médecine et 
du côté de l'anatomie comparée, de la morphologie, de la chimie « animale " ou "physiologique " aux prises avec l'identification des constituants élémentaires du vivant, que du côté de l'émergence de niveaux infra-organiques chez le vivant, tissulaire d'abord, puis cellulaire ${ }^{10}$. Ce sont les obstacles à l'apparition d'une science générale du vivant et les tâtonnements qui se traduisent dans les différents systèmes de classification en œuvre de la deuxième moitié du xviII siècle jusque dans le premier tiers du xIx ${ }^{e}$ siècle que cette étude veut explorer. Plutôt que de partir d'une conception a priori de la biologie, on a cherché à en situer les lieux putatifs et les signes, dans l'espace même des classifications assumées comme telles dans l'Encyclopédie, ou l'Encyclopédie méthodique, ou mises en œuvre dans d'autres institutions : autant que la mise en place des divisions des sciences dans des tableaux ou des prospectus, c'est leur " travail " dans le corps même des textes qui a retenu l'attention, c'est l'écart entre le statut assigné à une science dans un ordre encyclopédique, et ses usages dans le texte qui a paru suggestif, c'est enfin le jeu entre les exigences d'une unité du savoir, dont les différentes branches doivent être rattachées les unes aux autres, et la spécialisation croissante de chacune d'entre elles qui nous a intéressé.

LA CRISE DES CLASSIFICATIONS : DE L'ENCYCLOPÉDIE

À L'ENCYCLOPÉDIE METHODIQUE

Dans le "Système figuré des connaissances humaines " (voir supra, p. 18-19), l'étude des «sciences de la vie" - que nous prenons provisoirement pour périphrase désignant ce qu'à la fin du siècle un certain nombre d'auteurs nommèrent biologie - est doublement située, écartelée pourrait-on dire, entre ce qui relève de la mémoire, et ce qui relève de la raison, puisque la division des sciences se fait d'abord selon l'ordre des facultés de l'entendement, c'est-à-dire selon le point de vue du sujet connaissant. Ce n'est que secondairement que l'objet des connaissances fournit les moyens des divisions ultérieures. À la première, la mémoire, correspond une histoire naturelle, par opposition à une histoire sacrée, ecclésiastique, civile, ancienne et moderne : l'histoire naturelle elle-même

des sciences accessoires, Paris, Brosson, 1814, p. 424), ce qui correspondait à une conception mécaniste de la vie comme résultat de l'organisation. D'autres sens se font jour, en particulier chez Maupertuis, Buffon, Diderot, où organique devient synonyme d'animé ou vivant.

10. François Duchesneau, Genèse de la théorie cellulaire, Paris/Montréal, Vrin/Bellarmin, 1987. 
s'ordonne selon le regard que l'homme pose sur la nature, son uniformité, ses écarts, ses usages : ce n'est qu'après le troisième niveau hiérarchique de la classification, représenté par les accolades successives, que ce point de vue anthropocentrique sur la nature cède la place à une histoire des végétaux et des animaux, situés entre les minéraux en amont, et les éléments en aval, sans que l'on puisse déceler dans cette énumération un ordre de complexité croissante; si la catégorie des « écarts de la nature " englobe bien l'étude des monstruosités animales et végétales faisant ainsi pendant à celle de l'uniformité de la nature, les « usages de la nature " ne fournissent rien à l'histoire naturelle. Mais c'est à la philosophie qu'appartiennent, dans la «science de la nature", sous la rubrique "physique particulière", la zoologie et la botanique. Si l'on descend encore d'un étage dans cette hiérarchie classificatoire, on remarque que l'anatomie et la physiologie relèvent exclusivement de la zoologie et sont placées au même niveau que la médecine, l'art vétérinaire, le manège, la chasse ou la pêche, tandis que la botanique comprend seulement l'agriculture et le jardinage; cette pauvreté du domaine de la botanique, dont les objets semblent limités à l'utilité qu'ils ont pour l'homme, traduit bien les raisons historiques qui ont amené à cultiver cette science : nomenclature et étude des vertus ou des propriétés des plantes, dans la perspective d'un enrichissement de la matière médicale, subordination de la botanique à la médecine, qui expliquent à la fois qu'elle ait été étudiée sans interruption et que la définition de ses objets soit plus étroite que la zoologie. C'est tout le problème de la valeur du critère d'utilité dans la constitution d'une science qui se pose ici, et en définitive, des différents sens de l'utilité : D'Alembert, puis Condorcet s'élevèrent l'un et l'autre contre une conception trop étriquée et trop immédiate de l'utilité des sciences ${ }^{11}$, arguant que l'approfondissement des connaissances était en lui-même utile, en dehors de ses applications pratiques. Par ailleurs, la naturalisation de l'homme figure expressément dans le "Système figuré ", puisque la médecine et ses subdivisions sont incluses dans la zoologie, signifiant par-là que l'homme, « à ne considérer que la partie matérielle de son être ${ }^{12}$, fait partie du règne animal. Il ressort de cette première esquisse sur laquelle nous n'insistons pas ${ }^{13}$ que

11. Jean Le Rond, dit D'Alembert, Discours préliminaire de l'Encyclopédie, 1751, Paris, Gonthier, " Bibliothèque Médiations ", 1965 ; cette idée de Condorcet apparaît particulièrement dans les Éloges qu'il a prononcés.

12. Voir Bufron, op. cit. supra n. 7, t. II, chap. $1^{\text {er }}$, "Comparaison des animaux et des végétaux », p. 2 et le commentaire que Dideror fait de Buffon à l'article " Animal ", in Enc., t. I, 1751.

13. Voir sur ce point Michel Malmerbe, “ Mathématiques et sciences physiques dans le Discours préliminaire de l'Encyclopédie ", Recherches sur Diderot et l'Encyclopédie, 9, 1990, p. 109-146, et sa contribution dans ce volume, p. 13-37. 
la cohérence interne du "Système figuré " souffre quelques difficultés puisque les critères choisis pour définir les sciences ne peuvent être uniformément maintenus ou sont parfois contradictoires : l'exemple le plus probant est sans doute la distinction opérée entre la science de l'homme et la science de la nature, dans laquelle pourtant, par le biais de la zoologie, la connaissance de l'homme est réintroduite. La question de la place de l'homme dans la nature demeure controversée, et la solution trouvée renvoie à un dualisme du corps et de l'esprit que plusieurs articles de l'Encyclopédie s'efforcent discrètement de discuter.

En effet, on ne peut s'en tenir à la division des sciences proposée dans le "Système figuré ", tant la lecture des articles de l'Encyclopédie et du métatexte qui les accompagne et qui situe, en italique et entre parenthèses, leur place dans l'ordre encyclopédique, $y$ introduit d'écarts et de variations. Sans chercher à étudier de façon exhaustive cette distance, on peut néanmoins repérer trois aspects sur lesquels l'écriture des articles subvertit l'ordre du "Système figuré ": le chemin à parcourir concerne d'abord la réduction de l'écart entre histoire et science naturelle, c'est-àdire l'effacement de l'opposition entre une activité purement descriptive et classificatoire, mettant au premier plan la nomenclature, et une activité cherchant à se fonder sur la rationalité de ses principes et de sa méthode. L'ordre historique de l'acquisition des connaissances, decrire, nommer, classer, ne peut être tenu pour le modèle de développement de la science ni même pour son ordre d'exposition. Dans la redistribution des priorités qui s'opère, l'introduction croissante d'un point de vue fonctionnel aboutit aussi à une redéfinition de la science de la nature et, simultanément, à une redéfinition des objets de la botanique et de la zoologie. En second lieu, la distinction traditionnelle des règnes se trouve déplacée au profit d'une opposition entre vivant et non vivant, qui renforce l'inclusion de l'homme dans le règne animal, et la considération du végétal comme forme vivante. Enfin, la différence entre science fondamentale, pure, et ses applications, sans cesser de se manifester, fournit moins clairement la matière des divisions de la classification, tant il est vrai que la dignité croissante de la technè suppose la reconnaissance du savoir qu'elle exprime.

TEXTE ET METATEXTE DANS L'ENCYCLOPEDIE

Dans l'Encyclopédie, les entrées qui concernent les sciences en général ne sont pas toujours les plus explicites : par exemple, l'article «Zoolo- 
gie », beaucoup plus succinct que l'article « Botanique », se borne à évoquer la nécessité d'introduire des divisions parmi les animaux, en raison de leur immence diversité : la zoologie demeure une entreprise fondamentalement classificatoire. L'article «Physiologie " ${ }^{14}$, considérée comme une partie de la médecine, expose ce qu'est un traité de l'usage des parties, au sens galénique, ou un traité d' " œconomie animale ", et ce n'est que sous cette entrée, pourtant classée en médecine, que l'auteur s'élève à la considération des fonctions communes à tous les animaux, quadrupèdes, poissons, reptiles, oiseaux ${ }^{15}$. La physiologie, qui se propose l'étude des fonctions du vivant, reste prioritairement le domaine de l'homme, ne fait que s'ébaucher pour le règne animal, et laisse de côté le règne végétal. L'article "Anatomie » distingue une anatomie simple ou humaine et une anatomie comparée, qui concerne à la fois les brutes et les végétaux, et dont la finalité est subordonnée à la connaissance du corps humain. D'ailleurs, de Jaucourt, dans le bref article « Zootomie " qu'il donne comme synonyme d'anatomie comparée, déclare que son utilité est fort médiocre, et son intérêt de pure curiosité ${ }^{16}$. L'anatomie comparée consiste à s'occuper de la « recherche et de l'examen des différentes parties des animaux considérées relativement à leur structure particulière et à la forme qui convient le mieux à leur façon de vivre et de satisfaire à leurs besoins $" ~ "{ }^{17}$. Malgré l'élargissement esquissé au début de l'article, l'anatomie végétale n'est pas mentionnée comme telle. Cependant, si l'unification du monde vivant par-delà la distinction des règnes semble absente dans les quelques entrées qui viennent d'être examinées, et sont en cela conformes au «Système figuré », d'autres articles remettent en question cette classification, sans que l'on puisse d'ailleurs invoquer une quelconque raison chronologique. L'article « Animal », par exemple, qui est, comme on le sait, un commentaire de Diderot à partir d'extraits de Buffon, tirés du « Discours sur la nature des animaux » et de la "Comparaison entre les animaux et les végétaux » ${ }^{18}$, dégage les traits

14. Enc., t. XII, p. 537-538; l'article n'est pas signé, mais exprime une conception mécaniciste du vivant; par les références à Boerhaave et à Haller, fréquentes sous la plume de De Jaucourt, l'article pourrait être de ce dernier, qui a signé l'article suivant, mais ce n'est qu'une supposition.

15. Enc., t. XI, p. 360 a. L'article est signé «m », Ménuret de Chambaud, un médecin vitaliste de Montpellier.

16. Enc., t. XVIl, p. 744 a ; sur les transformations des articles « Anatomie " et « Physiologie " entre l'Encyclopédie, le Supplément et la Méthodique, et particulièrement sur l'histoire des disciplines, voir Roselyne REY, "Les métamorphoses de l'Encyclopédie : le cas des sciences de la vie ", Recherches sur Diderot et l'Encyclopédie, 12, 1992, p. 41-57.

17. Enc., article " Anatomie », t. I, p. 416 a.

18. Enc., article « Animal ", t. I, p. 472 a : " Cet examen nous conduit à reconnaître évidemment qu'il n'y a aucune différence absolument essentielle et générale entre les animaux et les végétaux." " 
communs à cette science générale du vivant. L'article " Botanique ", dû à Daubenton, s'élève également au-delà d'une conception de la discipline qui se limiterait à classer les espèces. L'un et l'autre article, concédant que ce sont les objets d'étude qui constituent les divisions de l'histoire naturelle, constatent que celles-ci ne sont pas toujours aisées, "puisque les sciences ne sont pas si distinctes qu'elles n'aient des rapports entre elles $"{ }^{19}$. La spécialisation très ancienne des disciplines, botanique et zoologie, est reconnue, mais la valeur de cette distinction est en même temps soumise à interrogation. La division en règnes est soigneusement notée «bien que les animaux et les végétaux [aient] beaucoup plus de rapports les uns aux autres qu'ils n'en ont aux minéraux $"{ }^{20}$, ne serait-ce qu'à cause de leur composition. Si le travail du naturaliste, au sens le plus général, consiste à établir les rapports que les objets ont entre eux, les différences et les ressemblances qui se trouvent entre les productions de la nature ${ }^{21}$, alors il faut bien mettre en valeur ce qui unit profondément animaux et végétaux, monde du vivant, par opposition au monde des corps bruts. Cette répartition binaire des productions de la nature entre en concurrence avec la division en trois règnes. Le même article "Botanique " propose de cette discipline une définition qui regroupe les deux aspects de l'histoire et de la science de la nature. Le métatexte leur assigne une place dans la philosophie, sous la physique particulière, mais le corps de l'article la définit comme " une partie de l'histoire naturelle qui a pour objet la connaissance du règne végétal tout entier "; à ce titre, la botanique comprend non seulement la nomenclature des plantes, leur culture et leurs propriétés, mais l'étude de la végétation (accroissement et développement des germes des plantes), base de toutes les autres parties. La "science de l'économie végétale " qui, comme le dit l'article "Botanique ", est " le plus difficile et le plus important », ou encore " le but le plus élevé " de cette science, fait ainsi pendant à "l'œconomie animale " : elle suppose une anatomie végétale qui aille jusqu'aux parties les moins apparentes ainsi qu'un intérêt pour les différentes activités vitales des végétaux : développement des germes, succion des racines et des feuilles, cours et évaporation de la sève; par-là, les objets d'étude de la botanique renvoient bien aussi à la physique générale. Dans cette approche qui relève de la physiologie végétale sans en porter encore le nom, et qui a été préparée par les travaux, entre autres, de Charles Bonnet Sur l'usage des feuilles, de Duhamel du Montceau sur la croissance des bois, de Fougeroux et plus tard de Mirbel, l'analogie s'impose entre

19. Enc., article «Botanique ", t. III, p. 226 a.

20. Ibid.

21. Ibid., p. 229 b. 
les fonctions de l'animal et du végétal : n'y a-t-il pas un rapport entre la formation des os par (ou dans) le périoste et celle du bois, entre la sève et le sang, entre les racines et la bouche, et ne peut-on parler dans les deux cas de digestion, de sécrétions et d'évacuation?

Ainsi, à notre sens, certains articles de l'Encyclopédie remettent en question la séparation de la botanique et de la zoologie, de l'histoire et de la philosophie, à travers deux questions : qu'est-ce qui sépare le règne animal du règne végétal ? Qu'est-ce que connaître le végétal ? Est-ce seulement le décrire, le distinguer et le nommer, ou est-ce aussi comprendre son « économie", sans subordonner cette recherche aux problèmes des familles, des genres et des espèces?

\section{L'ENCYCLOPEDIE METHODIQUE : UNE CRISE DE LA CLASSIFICATION}

Le problème des frontières entre les disciplines revêt une importance plus grande dans l'Encyclopédie méthodique que dans l'Encyclopédie puisqu'il s'agit, d'après le Prospectus de 1782, de remédier à la confusion des objets née de l'ordre alphabétique et du désir de « renfermer toutes les connaissances humaines dans un seul et même Dictionnaire, au lieu de donner à chaque science, à chaque art, son Dictionnaire $"{ }^{22}$. La fonction des Discours préliminaires en tête de chacun de ces dictionnaires est à la fois de délimiter les disciplines, de justifier les coexistences inévitables, par exemple entre physique et chimie, et de suggérer un mode de lecture du dictionnaire qui permette de le considérer comme traité ou manuel élémentaire. Malgré ces précautions, il est clair que la Méthodique met à jour une crise de la classification des sciences plutôt qu'un ordre méthodique ${ }^{23}$, et l'intervalle très long entre les premiers et le dernier volume ne fait que l'accentuer. Cette crise se joue sur deux plans : d'une part, l'impossible arbitrage entre les dictionnaires dont les sujets se chevauchent : c'est en particulier le cas pour la série consacrée à l'Histoire naturelle des animaux, qui contient aussi une « Histoire naturelle de

22. Encyclopédie méthodique ou par ordre de matière. 1782-1832, Prospectus et Catalogues (Réimpression photographique), 2 vol., Paris, Paul Jammes, 1964, t. 1, p. 6. Sur la Méthodique, voir Suzanne Tucoo-Chala, Charles-Joseph Panckoucke et la librairie française, 1726-1798, Pau/Paris, Marrimpouey jeune/Touzot, 1977; Robert Darnton, L'Aventure de l'Encyclopédie. Un best-seller au siècle des Lumières, Paris, Perrin, 1982.

23. Ce problème a fait l'objet d'un séminaire que j'ai donné au Centre Alexandre-Koyré (Paris) en 1990, et de plusieurs articles, en part. celui de Kathleen Dorg, " L'Encyclopédie méthodique et l'organisation des connaissances ", Recherches sur Diderot et l'Encyclopédie, 12, 1992, p. 59-69, et dans le même numéro, R. REY, « Les métamorphoses de l'Encyclopédie : le cas des sciences de la vie », p. 41-58. 
l'homme ", rédigée par Daubenton, et qui entre en concurrence avec les treize volumes de la partie médicale de la Méthodique, et surtout avec les volumes du Dictionnaire d'anatomie et de physiologie, devenu Système anatomique, qui est un ouvrage d'anatomie comparée. Le découpage en dictionnaires et l'écart entre les sujets annoncés et le contenu effectif des sujets traités, lui-même en évolution au cours de l'édition, révèle les hésitations sur les critères de définition d'une science : par son objet, par ses méthodes, ou par son point de vue? Sur le second plan, c'est plus précisément la hiérarchie qu'il convient d'établir entre une classification selon l'objet - qui retrouve alors, au sein de l'histoire naturelle, la division habituelle entre zoologie (et science de l'homme) et botanique - et une classification selon le point de vue statique ou anatomique, dynamique ou physiologique, qui transcenderait la distinction des règnes. Sans donner de solution à ce problème, la Méthodique accentue l'importance d'un point de vue physiologique.

Lorsqu'en 1782, Louis Daubenton rédige l'Avertissement et l'Introduction à l'Histoire naturelle des animaux, il est confronté à la délimitation du champ de l'histoire naturelle, " par rapport aux sciences qui ont le plus de rapports avec elle : telles sont l'Anatomie, la Matière Médicale, la Botanique, la Culture des plantes, la Chymie, la Métallurgie, etc. ${ }^{24}$, et il est amené en même temps à donner « les motifs qui ont déterminé à faire des dictionnaires particuliers non seulement pour chaque règne mais aussi pour chacun des ordres ou grandes classes des productions de la nature $"{ }^{25}$. En s'efforçant de circonscrire les limites de l'histoire naturelle, Daubenton ne se contente pas d'éliminer la connaissance de l'air, des météores, etc. - l'histoire naturelle redoublerait alors la physique pour ne garder que "la terre considérée dans les parties qui la composent et dans les êtres vivants qui l'habitent ${ }^{26}$, il cherche à la définir par les méthodes d'investigation qui sont les siennes : l'histoire naturelle s'intéresse selon lui aux objets "dans l'état de nature ", selon une attitude d'observation ou de contemplation, à la différence des sciences qui comme l'anatomie ou la chimie mêlent les procédés de l'art, interviennent sur les objets et les modifient. Cuvier reprochera durement à Daubenton cette séparation entre anatomie et histoire naturelle, acceptant d'ailleurs l'équivalence implicite posée par Daubenton entre analyse anatomique et analyse chimique et plaidera dans le Dictionnaire des

24. Encyclopédie méthodique. Histoire naturelle des animaux, Paris, Panckoucke, 1782, t. I, Avertissement, p. V.

25. Ibid., Avertissement, p. VI; les six dictionnaires prévus concernent les quadrupèdes vivipares et les cétacés, les oiseaux, les quadrupèdes ovipares et les serpents, les poissons, les insectes et les vers.

26. Ibid., p. XI. 
sciences naturelles en 1816 pour le rapprochement entre ces branches du savoir ${ }^{27}$. Il s'agit pour Cuvier d'établir une analogie, un rapport entre la conformation externe des animaux ou des plantes, et leur structure intime. À ses yeux, Daubenton manque ce rapport entre l'intériorité et l'extériorité, condition d'élaboration des lois de subordination des caractères et de corrélation des organes définies par Cuvier, et il accorde peu de place à l'anatomie comparée, ce qui disqualifie sa démarche. Cuvier remarque pourtant que Daubenton a été l'un des naturalistes les plus préoccupés de développer l'anatomie comparée des quadrupèdes et ne parvient pas à comprendre ce désir d'autonomisation de l'histoire naturelle autrement que par entêtement et préjugés.

Pourtant, à regarder de plus près l'Introduction de Daubenton, on constate que l'autonomisation de l'histoire naturelle par rapport à l'anatomie a pour contrepartie une unification à un autre niveau : quel que soit le règne considéré, le travail du naturaliste est d'observer la naissance, le développement et le dépérissement des corps ainsi que leurs métamorphoses éventuelles. C'était mettre au premier plan une histoire non plus au sens de description, mais au sens de processus inscrit dans le temps, caractéristique du vivant, et, par métaphore seulement, applicable au minéral. Cela impliquait en particulier la recherche des mécanismes de la génération et des différentes phases de développement de l'embryon animal ou végétal, tout en restant au niveau des individus. Daubenton désignait aussi explicitement l'objet le plus intéressant de l'histoire naturelle, l'économie animale ou végétale, c'est-à-dire le vivant considéré du point de vue de ses lois et de l'interdépendance de ses fonctions. Ce point de vue physiologique sur les règnes n'éliminait pas la tâche classique du naturaliste, opérer des distinctions dans les diverses productions de la nature, et nourrissait des réflexions sur le caractère arbitraire des systèmes de classification des objets naturels. Daubenton dénonçait clairement l'illusion d'une méthode qui présenterait « un tableau de la nature » : « toute méthode n'est qu'une invention de l'art " ${ }^{28}$. Il reprenait bien, à la manière de Buffon, les caractères distinctifs entre les trois règnes :

" Les minéraux diffèrent des plantes et des animaux, en ce qu'ils n'ont point d'organisation ni de vie. Quoique les plantes soient organisées, elles n'ont point de mouvement spontané ni de sentiment comme les animaux ${ }^{29}$,

27. Dictionnaire des sciences naturelles dans lequel on traite méthodiquement des différents êtres de la nature considérés soit en eux-mêmes d'après l'état actuel de nos connaissances, soit relativement à l'utilité qu'en peut retirer la médecine, l'agriculture, le commerce et les arts, 61 vol., Strasbourg, Levrault; voir l'Introduction de G. CuvieR, t. I, 1816, p. XXI.

28. Louis DaUBENTON, Introduction, in op. cit. supra n. 24, p. V.

29. Ibid., p. II. 
mais il ne s'étendait guère sur ce point; en revanche, il faisait de la notion d'individu et de son rapport à l'espèce l'instrument de délimitation entre le vivant et le minéral. Reprenant encore la formule buffonienne selon laquelle il n'y a que des individus dans la nature et pas d'espèces, il en détournait le sens en l'approfondissant :

« Il n'y a réellement et distinctement que des individus parmi les végétaux et les animaux. Les collections d'individus semblables les uns aux autres composent les espèces. Les caractères spécifiques, c'est-à-dire ceux qui distinguent les espèces sont essentiels aux individus : ils ne dépendent pas de la volonté du naturaliste, ils sont invariables dans la succession des générations et par conséquent de la Nature ${ }^{30}$.

La différence essentielle entre le minéral et le vivant provient justement de l'absence, dans le règne minéral, de tout principe d'individuation : « Parmi les minéraux, il n'y a point d'individus, et par conséquent, point d'espèces " ${ }^{31}$. Une lecture rétrospective de Daubenton a retenu plutôt l'affirmation de l'existence d'espèces dans la nature, et de leur invariabilité, alors que l'aspect le plus remarquable de cette pensée est autre : l'émergence d'une pensée biologique suppose la notion d'individualité. Dans le moment même où Daubenton se penche sur les problèmes de la classification (classification des sciences autant que des objets de l'histoire naturelle), il élabore la notion d'individualité biologique et retrouve les problèmes de génération et de développement qu'il avait au préalable définis comme objet d'observation. La perspective de la classification se dissout dans celle de la connaissance des productions naturelles. Sans être indifférent aux problèmes de nomenclature, Daubenton demandait que « le nom d'une chose » donnât quelque idée de sa " nature », et malgré le désir d'une langue commune pour la science, se prononçait pour le maintien du statu quo et l'emploi de la langue vernaculaire.

Comment dès lors concilier cette conscience émergente de l'unité du monde vivant, à travers son histoire, à travers le concept d'individualité, avec la pratique de dictionnaires séparés dans la Méthodique? En examinant, dans son Introduction, les trois règnes de la nature, Daubenton revenait sur la justification de ces divisions. Son analyse de la distinction entre minéraux ou corps bruts renforçait encore l'importance de la notion d'histoire individuelle, déjà mentionnée. Il désamorçait également par avance les possibles confusions entre chimie "animale » ou végétale et biologie; ce n'est pas parce que le charbon et les bitumes viennent des

30. Ibid., p. III.

31. Ibid. 
végétaux qu'ils appartiennent au même règne; les fossiles appartiennent aux corps organisés tant qu'ils conservent des vestiges d'organes. Et ce n'est pas parce que certaines substances se retrouvent dans les règnes animal et végétal qu'elles ont des analogies avec eux :

" l'huile n'est pas un individu du Règne animal et végétal : elle n'existe séparément de la substance des animaux et des plantes qu'après en avoir été tiré par des opérations de l'art ou par une décomposition de la Nature. Elle est originaire des corps organisés, mais elle n'a par elle-même aucun des caractères de l'organisation $"$ "32.

L'opposition entre l'état naturel et l'état produit par l'art devenait ici opératoire pour la différenciation entre le brut et le vivant, tout en changeant légèrement de signification : l'état naturel est pour le vivant un état spontané, un processus de transformation interne et autonome: "les corps organisés opèrent donc par eux-mêmes leur destruction : celle des corps bruts est indépendante de leur existence " ${ }^{33}$, elle est provoquée par des causes qui lui sont étrangères. C'est le processus de mort, et non de désagrégation, qui permet de tracer la limite entre le vivant et le minéral.

Le travail d'analyse mené par Daubenton concluait bien à une ligne de démarcation entre le vivant et le non-vivant, mais la question de l'absence d'êtres intermédiaires entre les règnes, qui pesait sur les débats et obnubilait littéralement les naturalistes avec le problème des zoophytes et des lithophytes, le conduisait à une séparation non moins brutale entre le végétal et l'animal. Certes, c'était plutôt une différence de degré (degré de composition, multiplication des organes et nombre de propriétés) qui distinguait le règne animal et le règne végétal. Mais, faute d'instruments conceptuels pour les distinguer, et en l'absence d'une vision claire de ce que pourrait être une "science des corps organisés ", Daubenton proposait une méthode de comparaison plus intuitive et plus phénoménale qu'analytique, qui devait à ses yeux fonder définitivement la différence :

«Il faudrait comparer les végétaux qui ont le plus d'organes avec les animaux qui en ont le moins. De cette manière, la question serait bientôt décidée : on ne trouverait guère d'analogie entre les arbres qui sont les plantes les plus organisées, et les vers qui sont les animaux les moins organisés. Les naturalistes qui ont cherché des êtres intermédiaires entre les animaux et les végétaux, ont suivi une autre méthode, qui renverserait l'ordre direct de production de la nature s'il existait. Ils ont indiqué une liaison du Règne végétal

32. Ibid., p. XII.

33. Ibid., p. XIII. 
et du Règne animal, par des rapports entre des végétaux et des animaux qui sont le moins organisés ${ }^{34}$.

En fait, les raisons pour lesquelles Daubenton récuse cette liaison sont de deux ordres; le premier est factuel et tend à montrer que les prétendus zoophytes, notamment polypes et coraux, sont bien des animaux, comme l'ont montré les travaux d'Ellis, et qu'on ne peut tirer argument de leur mode de reproduction pour en faire des végétaux. Le second est d'ordre logique : s'il y avait des êtres intermédiaires entre les règnes, on en trouverait à plus forte raison entre les différentes classes d'animaux et de végétaux. Or il n'y a pas de liaison entre quadrupèdes et oiseaux, la chauve-souris n'est pas l'intermédiaire entre les classes. En général, la découverte d'animaux nouveaux, loin de créer des maillons intermédiaires entre les classes, peut au contraire obliger à en créer de nouvelles. Parce que la représentation que Daubenton se fait de la Nature est opposée à la chaîne des êtres, parce qu'il s'affirme partisan résolu des interruptions, il ne peut arriver à penser le vivant dans son unité. C'est seulement entre les espèces que les nuances sont insensibles, la division des règnes, elle, est parfaitement fondée : « S'il n'y avait point d'interruption dans la suite des productions de la Nature, on n'aurait jamais eu l'idée de les distribuer par Règnes, par classes et par genres ${ }^{35}$.

Il y a bien des présupposés chez Daubenton, non des préjugés comme le dit Cuvier : présupposés d'un mode de connaissance dérivé directement de l'observation de la nature, d'une division en règnes, classes et ordres fondée dans la réalité de la nature et non dans l'esprit du naturaliste, présupposé aussi sur le faible degré d'organisation des vers, que les travaux de Lamarck et de Cuvier ont complètement modifié. Enfin, le cadre conceptuel de Daubenton demeure lié à la discussion sur les êtres intermédiaires aux confins des règnes, ce qui constitue une fausse piste, plutôt qu'à l'unité du vivant. Dans la constitution progressive d'une biologie, on voit bien qu'il n'y a pas de progrès linéaires, continus et nécessaires : le thème de la chaîne des êtres n'empêche pas Buffon de sceller la division du vivant et du non-vivant, tandis que le refus de cette chaine ininterrompue conduit Daubenton à instaurer des séparations qui interdisent de penser, globalement, à une science des corps organisés; ce qui a été gagné à travers le concept d'individualité biologique disposant d'une histoire, reste sans lendemain immédiat.

Un des préalables à cette vision d'une science des corps organisés, ou encore science physiologique, comme le dira Julien-Joseph Virey à

34. Ibid.; le passage que nous mettons en italique montre bien que Daubenton n'est pas étranger à la problématique de l'histoire ou de l'origine des corps organisés.

35. Ibid., p. XV. 
l'article « Histoire naturelle " du Déterville ${ }^{36}$, est le renforcement d'un point de vue physiologique concernant les végétaux. Le Discours préliminaire de Lamarck au Dictionnaire de botanique de la Méthodique, publié en 1783, insiste précisément sur ce point. Divisée en six parties qui comportent évidemment nomenclature, culture, connaissance des rapports et des affinités des végétaux, la botanique doit être fondée surtout sur "la physique des végétaux ", qui est à la fois une anatomie et une physiologie ; et Lamarck signale qu'avant de s'occuper de chaque plante en particulier, il importe de connaître « la nature des végétaux en général ", les processus de nutrition, déperdition, reproduction. C'est le terme de végétation qui s'impose pour regrouper et définir ces processus. Lamarck confirme donc la hiérarchie interne du savoir qui apparaissait déjà dans l'Encyclopédie, c'est-à-dire l'importance d'une anatomie et d'une physique végétale : physique toutefois, et non encore physiologie végétale, et l'analyse des rubriques montre bien que cette discipline ne s'est pas encore suffisamment individualisée.

NAISSANCE DE LA BIOLOGIE : ATTENTES ET OBSTACLES

Aux alentours de 1797, en Allemagne d'abord où l'on rencontre sans doute l'une des premières occurrences du mot chez Roze ${ }^{37}$, avant qu'elle ne soit chez Treviranus, en Angleterre où Erasme Darwin emploie le terme de "zoonomie ou lois de la vie organique" en un sens qui nous semble assez proche de biologie, se dessine l'exigence de traiter le monde vivant dans son unité : bien qu'Erasme Darwin inscrive d'abord son projet de " réduire tous les faits appartenant à la vie "animale " en classes, ordres, genres et espèces " dans une perspective médicale, le traitement qu'il en fait consiste à analyser les lois du mouvement de la matière, et à distinguer trois sortes de mouvements :

"The primary motions of matter may be divided into three classes, those belonging to gravitation, to chemistry and to life; and each has its pecular laws. [...] The third class includes all the motions of the animal and vegetable world; as well as those of the vessels, which circulate their juices, and of the

36. Nouveau dictionnaire d'histoire naturelle appliqué aux arts, à l'agriculture, à l'économie rurale et domestique, à la médecine etc., 36 vol., Paris, Déterville, 1816-1819; voir l'article " Histoire naturelle ", t. XIV, 1817, p. 542-564, en part. p. 555 ; la concurrence linguistique entre physiologie et biologie est ici aisément perceptible.

37. C'est Gehrard Müller qui a fait cette trouvaille, voir " First Use of "Biologie " ", Nature, 301, 1983, p. 744. 
muscles, which perform their locomotion, as those of the organs of sense, which constitute their ideas $"{ }^{38}$.

L'objet de la Zoonomie est de faire connaître les lois qui gouvernent tous les corps organisés en partant des plus simples, pour aller jusqu'à l'homme. La recherche d'un niveau élémentaire commun à tous les êtres vivants se fait chez Darwin par la mise au jour d'un mouvement primitif de la matière animale ou végétale : mouvement sensoriel qui transcende le clivage entre irritabilité et sensibilité, mouvement qui tire son origine du nerf, mais qui est répandu partout dans le corps. Tout être vivant est capable de réagir aux objets qui lui sont extérieurs, par une contraction, un changement de forme, un mouvement ou une configuration différente des fibres, qu'il nomme indifféremment « mouvement sensuel ou idée ». Prolongées au-delà, ces remarques pourraient conduire à analyser la théorie de la perception et de l'instinct chez les végétaux comme les animaux, ou, comme le dit le traducteur, mettrait en évidence la « liaison entre physiologie et métaphysique ". L'unification entre animal et végétal se fait aussi à partir d'une théorie de la génération : l'embryon a pour origine une sorte de filament vivant doué de diverses formes d'irritabilité et de sensibilité, qui se transforme, s'accroît et se différencie par épigénèse : au lieu que le mode de reproduction végétale par bourgeonnement soit considéré comme une forme dégradée de la génération, c'est lui au contraire qui fournit le modèle de la reproduction : végétaux et animaux se forment graduellement et se perfectionnent en fonction des exigences de lutte pour l'air et la lumière, qui finissent par faire naître de nouveaux comportements et de nouvelles parties. Il n'y a pas à proprement parler de nouvel individu créé, mais l'animal est comme une branche ou une élongation de ses parents. Ce qui importe ici, c'est à la fois l'existence d'un point de vue physiologique sur le vivant, et la recherche d'un niveau élémentaire commun, le filament vivant, qu'on ne peut lire décidément comme une traduction de la notion de fibre, mais qui est la plus petite forme organisée susceptible de transformation et d'échanges avec le monde extérieur.

Notre propos n'est pas de faire une étude de tous les textes fondamentaux qui conduisent progressivement d'une histoire naturelle à une biologie, qui excéderait évidemment les limites d'un article et dont certains aspects, plus spécialement tournés vers la problématique de la clas-

38. Erasmus DARWIN, Zoonomia or the Laws of Organic Life, 2 vol., Londres, J. Johnson, 1794-1796, t. 1, p. 5-6; sur ce point et sur la diffusion en France de ses idées avant la traduction française de Klyskers (1810-1811), voir R. REY, "Erasme Darwin et la théorie de la génération », in Histoire, nature et société, op. cit. supra n. 7. 
sification des espèces, ont déjà été abordés ${ }^{39}$, mais simplement d'indiquer ici quelques jalons pour comprendre la redistribution de la classification des sciences. Si l'intérêt des historiens, en raison du poids énorme de la problématique transformiste, a été surtout attiré par la question de l'origine et de la filiation des espèces, il nous semble que la constitution de la biologie comme science autonome est venue d'abord d'une sorte de physiologie générale : il faudrait mettre au premier plan les travaux de Spallanzani qui, sans contenir de réflexion particulière sur la classification des disciplines, a d'emblée conçu son étude des fonctions, respiration et reproduction surtout, comme englobant toutes les classes d'êtres vivants, des infusoires à l'homme, et qui a fait de la comparaison de ces fonctions dans les différentes classes, une des bases de sa méthode expérimentale ${ }^{40}$. Par ses traductions en français de Spallanzani, comme par ses propres recherches de physique végétale ou ses expériences sur l'absorption par les tissus vivants, Jean Senebier constitue un autre de ces jalons ${ }^{41}:$ les différents travaux sur l'absorption (Senebier, Magendie, Fodera et Dutrochet), ont été particulièrement importants dans la mesure où ils tendaient à prouver, sur des bases expérimentales, l'unicité des lois de la nature et où ils remettaient en cause l'intervention d'un agent vital dans les phénomènes d'osmose ${ }^{42}$. Pour en rester plus directement au domaine français, c'est sans doute Lamarck qui a le plus nettement formulé l'existence d'un domaine propre à la biologie, transcendant la distinction entre animal et végétal. Dans les Mémoires de physique et d'histoire naturelle publiés en l'an V (1797), et en particulier dans le septième mémoire, Lamarck formule une «théorie des êtres vivants ${ }^{43}$ dont l'originalité est de n'être

39. Giulio BarsanT, " Le Immagini della natura : scale, mappe, alberi, 1700-1800 », Nuncius, Anno III, fasc. 1, 1988, p. 55-125.

40. Lazzaro Spallanzanl, Saggio di osservazioni microscopiche sul sistema della generazione de Signori di Needham e Buffon, Modène, Eredi di Bartolomeo soliani, 1765; Nouvelles recherches sur les découvertes microscopiques et la génération des corps organisés, ouvrage trad. de l'italien de M. l'abbé Spallanzani, par M. Needham, Londres/Paris, Lacombe, 1769.

41. Jean Senebier, Opuscules de physique animale et végétale, 3 vol., trad. de l'italien de M. l'abbé Spallanzani, Paris, Duplain, 1787; les expériences sur l'absorption sont consignées dans ses Rapports de l'air avec les êtres organisés ou Traités de l'action du poumon et de la peau des animaux sur l'air, comme celle des plantes sur ce fluide, Genève, 1803 ; sur la méthode de Spallanzani, voir Pericle di PIETRo, « La méthode de Spallanzani à travers sa correspondance ", Dix-Huitième Siècle, 23, 1991, p. 97-105.

42. Sur ce point, voir notamment John V. PICKSTONE, « Vital Actions and Organic Physics : Henri Dutrochet and French Physiology during the 1820 s ", Bulletin of the History of Medicine, 50, 1976, p. 191-212; John LESCH, Science and Medicine in France. The Emergence of Experimental Physiology 1790-1855, Cambridge, Harvard University Press, 1984, en part. chap. 9 ; mais Jean Senebier, qui était suisse et non français, n'occupe pour ainsi dire pas de place dans ces études.

43. J.-B. LAMARCK, Mémoires de physique et d'histoire naturelle, établis sur des bases indépendantes de toute théorie; avec l'exposition de nouvelles considérations sur la cause générale 
fondée ni sur le recours aux propriétés vitales, pourtant florissantes à cette époque, ni sur une anatomie transcendante, qui se développera un peu plus tard, mais plutôt sur la nature des actions qu'exercent les êtres vivants sur ce qui les environnent, en des termes qui relèvent de la chimie, à ceci près que ce ne sont pas les corps élémentaires qui doivent être trouvés pour tous les êtres vivants, mais les processus qu'ils accomplissent :

« Les êtres vivants qui sont doués de la vie, ont, eux seuls, la faculté, par le moyen des fonctions de leurs organes, de former des combinaisons directes, c'est-à-dire d'unir ensemble des éléments libres et de produire immédiatement des composés $"{ }^{44}$.

Cette capacité, ce mouvement organique est inscrit dans le temps : les mêmes êtres vivants qui ont le pouvoir de former des composés sont également soumis eux-mêmes à un processus de désorganisation et de destruction. La vie n'est pas un être particulier, mais un processus d'assimilation et de réparation s'opposant en permanence à un processus de destruction : considérée chez Lamarck indépendamment de tout principe vital, elle se ramène à un mouvement, mouvement d'intégration des molécules essentielles assimilées par intussusception, mouvement qui résulte de l'exécution des fonctions de respiration, circulation, sécrétion, nutrition et génération. Cette clarification du domaine de la " science des corps organisés " est particulièrement importante dans la mesure où la confusion entre propriétés vitales et fonctions fait l'objet de beaucoup de discussions, chez Vicq d'Azyr autour de 1780, puis chez Chaussier, Moreau de la Sarthe et Magendie. Dans les mêmes années (1801 et 1802), Lamarck, qui avait utilisé le terme "biologie " dans l'Hydrogéologie, y revient dans ses Recherches sur l'organisation des corps vivants, dans une sorte d'urgence et de crainte de ne pouvoir rédiger sa biologie $^{45}$. Cette nouvelle science ne peut exister sans se libérer d'une

des dissolutions; sur la matière du feu; sur la couleur des corps; sur la formation des composés; sur l'origine des minéraux et sur l'organisation des corps vivants, Paris, l'Auteur, Agasse, Maradan, an V, p. 249.

44. Ibid., p. 245.

45. J.-B. LAMARCK donne l'exposé des "motifs de cet ouvrage ", les Recherches sur l'organisation des corps vivants, Paris, l'Auteur, au Muséum d'histoire naturelle, Maillard, an X; nous utilisons la réédition, Paris, Fayard, 1986, p. 9-10: « Je fus en outre déterminé à l'étendre [Lamarck parle de son discours d'ouverture] en y employant des matériaux que je réservais pour ma Biologie; parce que, considérant que je suis extrêmement surchargé de travaux relatifs aux sciences physiques et naturelles, et remarquant néanmoins que ma santé et mes forces sont considérablement affaiblies, j'ai craint de ne pouvoir exécuter ma Biologie, à laquelle je ne projette de mettre la dernière main qu'après avoir publié mes observations sur la Météorologie, qui feront le complément de ma Physique terrestre "; dans ce texte, Lamarck met le terme de Biologie en italiques et renvoie à son Hydrogéologie. Sur Lamarck 
activité principalement classificatoire, qui a pesé sur les sciences naturelles, alors que leur direction devrait être toute différente. Dans le Discours d'ouverture du 27 floréal an X au Muséum, Lamarck compare l'activité du zoologiste et du botaniste à ce géographe qui s'encombrerait la mémoire du nom de tous les villages, lieux-dits et coteaux, et n'aurait plus le temps de connaître les différents climats du globe, la direction ou la nature des chaînes de montagnes, etc. C'est un changement d'orientation qu'il propose :

«Combien donc n'importe-t-il pas, pour le progrès et la dignité des sciences naturelles, de diriger nos recherches non seulement vers la détermination des espèces, mais encore vers la connaissance de l'origine, des rapports et du mode d'existence de toutes les productions naturelles dont nous sommes entourés ${ }^{46}$.

Dans la seconde partie des Recherches sur l'organisation des corps vivants, Lamarck développe l'ensemble de ces points, qui vont de la nature et de l'origine de la vie à la conservation des variations et au rôle de l'habitude dans la forme des organes, en passant par la spécialisation des fonctions dans la série des êtres vivants, selon des territoires délimités. On comprend dès lors pourquoi cette conception de la biologie a eu du mal à s'imposer et pourquoi, somme toute, elle est restée, pour ainsi dire, en attente de concrétisation disciplinaire. Ce n'est pas tant à cause de l'idée d'évolution que l'entreprise lamarckienne paraissait nouvelle. C'est parce que sa biologie impliquait, par exemple, la question de la génération spontanée, préjugeait d'un ordre de production des êtres vivants selon une histoire, allant du plus simple au plus complexe, c'est parce qu'il disqualifiait la pratique classificatoire de bien des naturalistes de son temps et parce que les conditions théoriques de cette science du vivant n'étaient pas réunies.

La concrétisation de ces deux aspects, point de vue physiologique et recherche d'un niveau élémentaire commun, se retrouve chez Treviranus dans sa Biologie oder Philosophie der lebenden Natur für Naturforscher und Aertze, dont le premier volume fut publié en 1802, mais elle est asso-

et le problème du vivant, voir Richard W. BuRCKHARD, The Spirit of System. Lamarck and Evolutionary Biology, Cambridge, Mass., Harvard University Press, 1977; Ludmilla J. JordANova, Lamarck, Oxford, Oxford University Press, 1984, en part. p. 44-57; Pietro Corsi, The Age of Lamarck. Evolutionary Theories in France 1790-1830, Berkeley, Los Angeles, University of California Press, 1988, en part. p. 62-75 ; Goulven LAURENT, Paléontologie et Évolution en France 1800-1860, de Cuvier-Lamarck à Danwin, Paris, Éd. du Comité des travaux historiques et scientifiques, 1987, p. 57-69.

46. J.-B. LAmarCK, "Discours d'ouverture du cours du 27 floréal an X au Muséum », in Recherches, op. cit. supra n. 45 , p. 13. 
ciée de surcroît à une claire conscience des obstacles à franchir à la fois par rapport aux pratiques traditionnelles de l'histoire naturelle et par rapport à la situation de cette nouvelle science. Toute l'introduction de sa Biologie est consacrée à signifier, d'une part, la clôture de ce qu'il appelle - peut-être un peu injustement - "l'ère linnéenne " et donc à sortir de l'ornière classificatoire, et, de l'autre, à essayer de situer la biologie par rapport à une classification des sciences. Les connaissances concernant la nature vivante ont longtemps été aussi isolées les unes des autres que des pyramides dans le désert d'Égypte. Pire même, elles ont été dispersées dans différentes ramifications de la connaissance humaine, et, le plus souvent, subordonnées à un principe d'utilité défini par les exigences de la médecine. Aussi la biologie ne peut-elle se constituer, selon Treviranus, que par une double émancipation (par rapport à l'histoire naturelle et par rapport à la médecine), ce qui équivaut à la fonder par un acte qui est à la fois de séparation et d'autonomisation dont la prétention explicite est d'accorder à la biologie un statut équivalent à celui de la physique pour les corps bruts, et un acte de réunification de connaissances éparses jusque-là dans différentes disciplines. Voici comment Treviranus définit, en 1802, l'objet et l'importance de la biologie :

«L'objet de nos recherches sera la diversité des formes et des phénomènes de la vie, les conditions et les lois selon lesquelles cet état de vie se produit, les causes par lesquelles la vie a lieu. Cette science qui s'occupe de ces objets, nous l'appellerons Biologie ou connaissance du vivant $"{ }^{47}$.

L'ensemble des textes que nous venons d'examiner indiquent assez clairement l'émergence d'une pensée biologique qui cherche à se situer et à se définir dans un arbre des connaissances humaines. Mais comme on le voit à travers le commentaire de Treviranus, il ne suffit pas pour en définir l'objet de parler de l'unité du monde vivant. Il existe, au début du $\mathrm{XIX}^{\mathrm{e}}$ siècle, une pluralité d'interprétations possibles de ce que peut être la biologie dans ses rapports avec les autres sciences et avec les méthodes : ia façon dont se recompose le paysage intellectuel dans différents contextes nationaux et les orientations que prennent cette biologie naissante peuvent expliquer bien des obstacles, des détours et des retards. Ainsi la conception de la biologie de Treviranus sera beaucoup plus facilement acceptée en Allemagne qu'en France pour un ensemble de raisons : impulsion donnée par Kant aux études sur le vivant à travers la formula-

47. Gottfried Reinhold Treviranus, Biologie oder Philosophie der Lebenden Natur für Naturforscher und Aerzte, Göttingen, Johann Friedrich Röwer, 1802, t. 1, Vorrede, p. V. 
tion du concept d'organisme ${ }^{48}$, orientation plus nette en faveur de la physiologie, comme le montre en particulier les programmes des universités, conception organique de l'unité du savoir défendue par la Naturphilosophie $^{49}$, qui permet de mieux faire le lien entre les différentes sciences de la nature, enfin choix d'une méthode synthétique qui se concrétise dans l'anatomie transcendante et la morphologie, là où en France s'instaure très tôt la spécialisation des savoirs, et le primat de la méthode analytique. Ce que révèle la Méthodique dans son ambition même de faire des dictionnaires séparés pour la médecine, l'anatomie et la physiologie, l'histoire naturelle des animaux découpée en classes, la botanique, c'est à la fois la sanction de l'explosion du savoir et de la spécialisation croissante, et l'aveu implicite de l'impossibilité, par rapport à l'Encyclopédie de Diderot et D'Alembert, de retisser les fils du savoir pour faire une science du vivant unifiée.

L'œuvre de Cuvier, et son évolution dans les toutes premières années de sa carrière, peut paraître assez révélatrice des difficultés d'installation de cette science unitaire du vivant qui en est encore à se chercher. Mais compte tenu du poids de Cuvier dans les institutions scientitiques en France, Académie des sciences, Muséum notamment, ses positions ont très rapidement acquis force de loi $^{50}$. L'Analyse des travaux de la classe des sciences mathématiques et physiques, rédigée chaque année et publiée dans les Mémoires de l'Institut, fournit une sorte d'état des lieux dans lequel Cuvier ne peut se dispenser de donner son point de vue : c'est peu à peu un véritable rapport de forces qui se constitue et dont on peut suivre les traces et les effets à travers les rubriques de cette histoire des sciences. Ainsi, après avoir semblé un moment proche de Bichat et de ses conceptions de la vie, dans ses Leçons d'anatomie comparée de 1801, Cuvier prend nettement position contre le recours au principe vital dans les sciences physiologiques, en indiquant en 1806, à l'occasion de la seconde édition des Nouveaux Éléments de la science de l'homme de Paul-Joseph Barthez:

" Pour avoir le droit d'en comparer l'emploi à celui de la gravitation universelle, il faudrait analyser séparément chaque phénomène de la vie; détermi-

48. Voir Timothy Lenorr, The Strategy of Life. Teleology and Mechanics in NineteenthCentury German Biology, 1982, Chicago, University of Chicago Press, 1989.

49. Sur ce point, voir Philosophies de l'université : l'idéalisme allemand et la question de l'université. Textes de Schelling, Fichre, Schleiermacher, Humboldt, Hegel réunis à l'initiative du Collège de Philosophie, prés. par Luc Ferry, Jean-Pierre Pesron et Alain Renaut et trad. de l'allemand par G. Goffin et al., Paris, Payot, 1979. La publication, dans le Magasin encyclopédique, des programmes des universités allemandes dans le domaine de la médecine et des sciences naturelles va dans le même sens.

50. Voir sur ce point Dorinda Outram, Georges Cuvier. Vocation, Science and Authority in post-Revolutionary France, Manchester, Manchester University Press, 1984. 
ner la part qu'y ont les lois de la physique et de la chimie ; comparer ensuite dans chaque phénomène les éléments que ces deux sciences auraient pu fournir à ceux qui seraient restés de même après l'analyse des autres phénomènes ; voir si tous ces éléments connus, extraits pour ainsi dire séparément des phénomènes divers, ont quelque chose de commun entre eux; rechercher enfin les lois qu'il faut attribuer à ce principe commun, si l'on trouve qu'il existe, pour qu'en le combinant avec ceux des sciences ordinaires, il donne de tous les phénomènes observés une explication satisfaisante pour la raison et fasse prévoir d'avance avec quelque exactitude les phénomènes qui devront arriver dans des circonstances nouvelles. C'est alors seulement que la physiologie pourra se flatter d'avoir un principe particulier comme l'astronomie en a un ${ }^{51}$.

Ce commentaire limpide qui rappelait les exigences de la science moderne, mettait l'accent sur l'importance de la méthode analytique, et dans le même passage de 1806, il esquissait une définition des " sciences intérieures » (chimie, anatomie comparée, physiologie), par opposition aux sciences extérieures. Ni du point de vue d'une philosophie générale du vivant, dont le principe vital ou les propriétés vitales auraient pu être le lieu d'unification, ni du point de vue de la méthode, la biologie au sens où elle était entendue dans les pays de langue allemande ne pouvait trouver sa place dans le contexte de la France impériale et cuviérienne. En 1795, Cuvier, dans un Discours prononcé à l'ouverture du Cours d'Anatomie comparée qu'il fait en remplacement du citoyen Mertrud le 15 frimaire an $I I I$, procédait à l'examen comparé des méthodes analytique et synthétique en histoire naturelle : s'il reconnaissait que seule la méthode analytique avait une valeur heuristique, tandis que la méthode synthétique ne pouvait servir qu'à exposer la science, une fois celle-ci acquise, il hésitait encore, et choisissait la seconde pour des raisons qui n'étaient pas seulement pédagogiques. À l'intérieur même de la démarche synthétique, qui a le mérite de fournir des principes généraux et communs, deux attitudes étaient encore possibles : ou bien « considérer chaque classe d'animaux et en décrire de suite tous les organes ", ou bien, " considérant chaque organe, de parcourir toutes les classes afin de découvrir les diverses modifications que cet organe reçoit $"{ }^{52}$. Pesant les mérites respectifs de chacune, Cuvier choisissait de nouveau la seconde, parce qu'elle permettait de satisfaire le véritable but de la zoologie, qui était selon lui l'adoption d'un point de vue physiologique : mais cette seconde méthode était-elle

51. Georges Cuvier, «Analyse des travaux de la classe des sciences mathématiques et physiques depuis le $1^{\text {er }}$ messidor an 13 jusqu'au $1^{\text {er }}$ janvier 1806 ", Mémoires de l'Institut, t. 7, 1806, p. 78.

52. ID., "Discours prononcé à l'ouverture du Cours d'Anatomie comparée, an III ", Magasin encyclopédique, t. 5, l'an quatrième (1795), p. 152. 
vraiment "synthétique "? ne s'agissait-il pas plutôt de sélectionner et d'isoler un organe pour en comprendre les transformations dans la série animale, c'est-à-dire de procéder à une analyse qui est le soubassement même de l'anatomie comparée? Certes, comme le disait Flourens dans son Éloge de Cuvier, cette méthode consistait à savoir tout ce qui est d'absolue nécessité pour chaque organe et tout ce qui peut lui être ajouté ou retranché et que révèle précisément la comparaison de cet organe à travers les espèces, les genres et les classes. Si le point de vue physiologique, introduit par Cuvier et concrétisé à travers les principes de subordination des caractères et de corrélation des organes, avait une indéniable valeur prédictive, qui assura d'ailleurs la gloire de Cuvier, il reste que la physiologie n'était pas étudiée pour elle-même, mais était mise au service de l'anatomie comparée : ni le Tableau élémentaire de l'Histoire naturelle des animaux de 1798, ni le Règne animal distribué d'après son organisation de 1817, ne dépassaient ce point de vue sur la place de la physiologie. En prétendant " faire marcher de front l'anatomie et la zoologie ", Cuvier cherchait à faire marcher de front « les dissections et le classement », cherchait, dans ses « premières remarques sur l'organisation [,] des distributions meilleures ${ }^{53}$. La méthode de travail demeurait analytique et le modèle celui de la chimie. Avant les Rapports à l'Empereur sur le progrès des sciences, des lettres et des arts depuis 1789. Chimie et sciences de la nature, les rubriques utilisées par Cuvier pour présenter l'histoire des travaux de sa classe demeuraient fluctuantes dans leur distribution et ne correspondaient pas toujours aux sections de l'Institut. La chimie se taillait la part du lion et se décomposait en chimie générale, chimie animale et chimie des corps gras. On voyait apparaître une catégorie « minéralogie et géologie " dans laquelle étaient consignées les recherches concernant les animaux fossiles, qui pouvaient aussi se trouver répertoriées en zoologie; la zoologie ne comprenait pas toujours une rubrique " anatomie ", ni « physiologie animale »; la botanique était parfois jointe à l'agriculture. Ces fluctuations indiquent que le territoire de chaque discipline n'a pas été clairement redéfini : coexistent une classification héritée, en gros, de l'Encyclopédie et fondée sur les objets d'étude, avec des découpages où les frontières entre anatomie et physiologie, physiologie et chimie, physiologie et histoire naturelle sont devenues mouvantes. C'est seulement en 1808 que le Bulletin des sciences de la Société philomatique remplace les catégories "physique des animaux et physique des végé-

53. ID., Tableau élémentaire de l'histoire naturelle des animaux, Paris, Baudouin, an VI, p. VI ; sur les premières années de Cuvier, voir R. REY, « La circulation des idées scientifiques entre la France et l'Allemagne : le cas Cuvier ", in Allemands en France. Français en Allemagne, 1715-1789. Contacts institutionnels, groupes sociaux, lieux d'échange, publ. par Jean Mondot, Jean-Marie VAlentin, Jürgen Voss, Francia, 25, 1922, p. 197-208. 
taux " par celles de "physiologie animale " et "physiologie végétale ", consacrant ainsi l'égalité de traitement entre les deux règnes, que le titre même de l'ouvrage de Senebier, Physiologie végétale, publiée en l'an VIII, et celui de Mirbel, Traité d'anatomie et de physiologie végétales, ont contribué sans doute à imposer ${ }^{54}$. Le Rapport à l'Empereur de 1809 n'homogénéise pas complètement ses propres rubriques : l'introduction ne mentionne que les très classiques découpages, anatomie, physiologie, zoologie et botanique; mais au début de la section " histoire naturelle", Cuvier introduit une différence en fonction du rapport entre l'observateur et son objet d'étude :

«... la différence essentielle entre les sciences générales et l'histoire naturelle, c'est que dans les premières, on n'examine [...] que des phénomènes dont on détermine en maitre toutes les circonstances, et que dans l'autre les phénomènes se passent sous des conditions qui ne dépendent pas de l'observateur " :

aussi la ligne de partage passe-t-elle entre "chimie ordinaire " et " chimie vitale " ${ }^{55}$. C'est peut-être moins le thème vitaliste de la variabilité des phénomènes vitaux qui est ici considéré, que la possibilité ou non d'en maitriser les circonstances et les variations, et la méthode qu'elle impose. Dans la composition d'ensemble du Rapport de 1809 , où sont juxtaposées des rubriques de niveaux très différents - les intitulés disciplinaires voisinant avec des objets particuliers, fossiles, géographie de la France -, Cuvier, après avoir traité de la minéralogie et de la géographie physique, définit une «Histoire naturelle des êtres vivants", qui comprend une "partie chimique », une «partie anatomique » et une " partie physiologique », à l'intérieur de laquelle se distingue une «physiologie générale » et une " physiologie particulière ». C'est cette " physiologie générale » dont les objets pourraient être considérés comme ceux de la biologie; le texte de Cuvier dans le Rapport nous paraît singulièrement embarrassé : son lyrisme contraste avec la sobriété des résultats et des définitions utilisés dans le reste du Rapport et ne correspond guère, quant au fond, aux propos de 1806 déjà mentionnés.

« Il nous reste à traiter de la partie dynamique du grand problème de la vie, ou des forces qui produisent les mouvements nombreux dont nous avons dit

54. J. Senebier, Physiologie végétale, 5 vol., Genève, an VIII ; Charles-François Brisseau de Mirbel, Traité d'anatomie et de physiologie végétales, ouvrage servant d'introduction à la botanique, 2 vol., Paris, F. Dufait, an X.

55. G. Cuvier, Rapports à l'Empereur sur le progrès des sciences, des lettres et des arts depuis 1789. II : Chimie et sciences de la nature, 1809, préf. de Denis Woronoff, prés. et notes sous la dir. d'Yves LaIssus, Paris, Belin, 1989, p. 135. 
qu'elle se compose. C'est en effet, s'en faire une idée fausse que de la considérer comme un simple lien qui retiendrait ensemble les éléments du corps vivant, tandis qu'elle est, au contraire, un ressort qui les meut et les transporte sans cesse : ces éléments ne conservent pas un instant le même état ni la même composition; plus sa vie est active, plus ses échanges et ses métamorphoses sont continuels [...] C'est ici que commence l'emploi raisonnable du terme forces vitales : pour peu que l'on étudie en effet les corps vivants, on ne tarde point à s'apercevoir que leurs mouvements ne sont pas tous produits par des chocs ou des tiraillements mécaniques et qu'il faut qu'il y ait entre eux une source constante productrice de force et de mouvement ${ }^{56}$.

Sans doute Cuvier parle-t-il de forces vitales, notion peut-être plus acceptable à ses yeux que celle de principe vital, parce qu'elle est fondée sur les propriétés des tissus et des organes; sans doute le vitalisme discret dont ce texte fait état est-il plus celui de Bichat que celui de Barthez; mais il semble bien que ce texte révèle tout d'abord le lien intime entre une philosophie vitaliste, passée de la médecine aux sciences naturelles, et une conception générale des êtres vivants, " histoire naturelle des êtres vivants » comme le dit Cuvier et plus précisément encore, " physiologie générale », dont les objets recouvrent les définitions ultérieures de la biologie : rien d'étonnant à cela puisque le vitalisme affirme la spécificité du vivant et des méthodes d'étude qu'il requiert, ainsi que l'autonomie de son étude par rapport à la physique et à la chimie. Mais, comme l'indique la critique de 1806, ainsi que toute l'orientation ultérieure des travaux de Cuvier, il ne paraît guère possible de fonder cette unité de la science des êtres vivants sur des propriétés ou des forces vitales; en 1809, François Magendie se livre à une critique plus décisive encore de cet envahissement par les propriétés vitales ${ }^{57}$. Quelles sont alors les solutions possibles pour fonder une biologie? L'une pourrait être de s'appuyer sur la méthode d'analyse, comme cela a été plusieurs fois esquissé dans les textes de cette période : mais l'analyse, qui regroupe dissection anatomique et «dissection chimique » délimite insuffisamment le territoire de la chimie vitale, par rapport à la physiologie générale, et d'autre part, par définition, fait passer son objet d'étude de l'état de vivant à son contraire : c'est vraisemblablement en fonction de cette impasse que la physiologie deviendra, en France, expérimentale et fondée sur la vivisection, seul moyen de maintenir la méthode analytique dans les sciences du

56. Ibid., p. 183-184.

57. François MAGENDIE, "Quelques idées générales sur les phénomènes particuliers aux corps vivants ", Bulletin des sciences médicales, 4, 1809, p. 145-170; sur ces questions, voir R. REY, Naissance et développement du vitalisme en France, thèse de doctorat de Paris I, 1987, à paraitre dans « Studies on Voltaire and the 18th Century », Oxford, Voltaire Foundation. 
vivant. Une autre possibilité aurait consisté à unifier ces différentes recherches sous la rubrique, que l'on trouve fréquemment dans les textes de cette époque, de "science des corps organisés" : mais tout le problème vient de ce que, dans le contexte vitaliste dont nous avons parlé, la vie n'est pas l'organisation, elle n'est pas même assimilable à l'organique : d'ailleurs la difficulté de classement des corps organisés fossiles dans les rubriques des périodiques, tantôt en zoologie, tantôt en botanique, tantôt en minéralogie et géologie, illustre bien cette différence entre l'organisé et le vivant: les Annales des sciences naturelles qui, dès leur parution en 1824, ont une rubrique "Anatomie et Physiologie animale, zoologie ", distincte d' "Anatomie et Physiologie animale, botanique ", finissent en 1828 par ajouter une mention spéciale à la troisième rubrique qui devient " Minéralogie, géologie, corps organisés fossiles ${ }^{58}$. La reconnaissance de l'unité du monde vivant ne peut, en ce tout début de $\mathrm{xIx}^{\mathrm{e}}$ siècle, se fonder ni sur la notion d'organisation, qui n'a pas encore atteint le niveau cellulaire, ni sur la méthode analytique, ni sur une philosophie de la nature : les hésitations déjà mentionnées chez Cuvier à propos du vitalisme deviennent de véritables répugnances s'agissant de la "philosophie de la nature" qui prévaut au même moment en Allemagne :

«ce projet imposant", dit Cuvier en évoquant la généralisation du concept d'organisation à l'échelle du grand Tout de la Nature, «ne s'est exécuté jusqu'à présent qu'en passant continuellement et brusquement, sans règle fixe, qu'en appliquant sans cesse un terme moral à un phénomène physique et réciproquement; qu'en employant des métaphores au lieu d'arguments : en un mot, cette méthode, qui n'a fait découvrir jusqu'à présent aucun fait nouveau auquel on n'ait pu arriver par la marche ordinaire, est telle que l'on a peine à concevoir la fortune qu'elle a faite dans un pays renommé par sa raison et sa logique et comment elle y a trouvé des partisans parmi des hommes d'un talent réel, et dont les expériences ont d'ailleurs enrichi les sciences de faits précieux... $\gg{ }^{59}$.

Comment peut-on être Naturphilosoph ? Cette perplexité de Cuvier, très perceptible dans la note qui accompagne le fragment que nous venons de citer va se transformer en hostilité déclarée dans les années qui suivent, et la controverse avec Geoffroy Saint-Hilaire ne pourra que

58. Annales des sciences naturelles comprenant la physiologie animale et végétale, l'anatomie comparée des deux règnes, la zoologie, la botanique, la minéralogie et la géologie, par MM. Audouin, Ad. Brongniart et Dumas, 1824-1833 ( $1^{\text {re }}$ série) : les " corps organisés fossiles " apparaissent dans le tome XIV; après 1833, les Annales se scindent en botanique et zoologie et prendront ultérieurement le sous-titre biologie animale et biologie végétale.

59. G. CUVIER, Rapports, op. cit. supra n. 55, p. 191. 
l'accentuer. Mais sans aller jusqu'à cette fameuse année 1830 et aux débats commentés par Goethe, il semble que se mette en place chez Cuvier, et sans doute plus généralement dans les milieux des naturalistes et physiologistes français, la conviction que ce projet d'unification du monde vivant qui a nom «biologie » en Allemagne et verse dans une vitalisation de la Nature entière, est fondamentalement contraire à la bonne méthode de la science, et comme on le dit alors, à « la saine philosophie ". Dès lors, l'idée de biologie et la philosophie de la nature allemande sont apparues comme liées, comme rattachées à une démarche philosophique spéculative. Dans un petit Tableau que nous reproduisons ci-dessous, Moreau de la Sarthe rattachait bien la physiologie générale à l'étude « transcendante » des caractères et des forces organiques, transcendante au sens où l'on parle d'anatomie transcendante, mais la connotation n'était pas neutre.

«Sciences anatomiques et physiologiques

ou Physique

Particulière des corps organisés,

Rapportés à deux genres principaux d'études; savoir

I Les études des opérations organiques

\begin{tabular}{l|l}
$\begin{array}{l}\text { Sciences } \\
\text { anatomiques } \\
\text { comprenant }\end{array}$ & $\begin{array}{l}\text { 1) l'anatomie intérieure ou } \\
\text { générale } \\
\text { 2) l'anatomie descriptive }\end{array}$
\end{tabular}$\left\{\begin{array}{l}\text { de l'homme et } \\
\text { des animaux } \\
\text { des plantes } \\
\text { de l'homme } \\
\text { (anthropographie) } \\
\text { des animaux } \\
\text { (anatomie comparée) } \\
\text { des plantes } \\
\text { (anatomie végétale) }\end{array}\right.$

II Les études des phénomènes organiques ou les sciences physiologiques divisées en sections principales

1) La physiologie générale ou l'exposition transcendante des caractères et des forces organiques

2) La physiologie parti- dans l'homme. Physioculière ou l'exposition analy- logie proprement dite tique des opérations orga- dans les animaux. Zooniques appelées fonctions nomie (ou physiologie comparée) dans les plantes. Physiologie végétale ${ }^{60}$.

60. Encyclopédie méthodique. Médecine, Paris, Agasse, t. XII, article « Sciences physiologiques » (signé Moreau de la Sarthe), 1827, p. 3 ; Moreau attire l'attention sur l'emploi du pluriel. 
Bien que Moreau ne mentionne pas la physiologie générale comme une étude synthétique, on voit comment dans le tableau, elle s'oppose à la physiologie particulière analytique. L'emploi du pluriel les « sciences physiologiques " témoigne de l'expansion du domaine désormais couvert par l'étude du vivant, et de la nécessité d'opérer de nouvelles divisions : l'opposition du statique et du dynamique, déjà présente au début du siècle se confirme, de même que l'opposition du général et du particulier. Il reste bien une place pour une physiologie s'élevant à la généralité des phénomènes de la vie, mais son " occupation » demeure controversée. En 1823, quand Michele Fodera avait publié son Discours sur la biologie ou science de la vie, la critique avait été particulièrement sévère : c'est que Fodera, qui pourtant avait travaillé en physiologie expérimentale sous Magendie, qui avait mené des travaux sur l'absorption, définissait précisément la biologie sur des bases qui pouvaient sembler spéculatives :

" La science de la vie, ainsi que de toutes les autres sciences naturelles ne peut être fondée que sur l'énergétique, qui dépend d'un premier fait, celui de la nature de notre intelligence. [..] L'introduction à la biologie sera l'exposé des principes de l'énergétique, qui est la base de toute science naturelle, de toute véritable philosophie, la science des réalités, la seule qui puisse anéantir le monde des chimères. L'étude des actions et de leurs propriétés fixe les limites des connaissances scientifiques; et des données de l'énergétique découle un tableau des connaissances naturelles, considérées d'après leur nature et leur filiation ${ }^{6}{ }^{6}$.

Mais cette " énergétique " avait bien peu à voir avec un concept physique ou physiologique : science des sciences, l'énergétique devait retrouver l'enchaînement ordonné, par ordre de complication croissante, des phénomènes physiques, chimiques et vitaux, en étudiant d'abord le « premier anneau des êtres vivants ", pour en arriver à une conception globale de l'énergétique de la vie et de l'énergétique de la Nature où, suivant l'expression hippocratique, " tout concourt, tout conspire, tout consent ». La biologie avec Fodera était plus que jamais spéculative.

Entre la fin du xviII ${ }^{e}$ siècle et la première moitié du $x x^{e}$ siècle, la question d'une science du vivant est à l'ordre du jour, et possède déjà un nom, sans avoir en quelque sorte son lieu épistémique dans la classification des sciences. À titre de repères, on peut signaler que, après cette première " naissance avortée " autour de 1800, le terme de biologie figure de manière sporadique dans les dictionnaires ou les traités : on lę trouve par exemple en 1827 dans le Dictionnaire des sciences naturelles, sous une

61. Michele Fodera, Discours sur la biologie ou science de la vie, Paris, Baillière, 1826. Voir la reproduction de ce tableau, p. 196. 


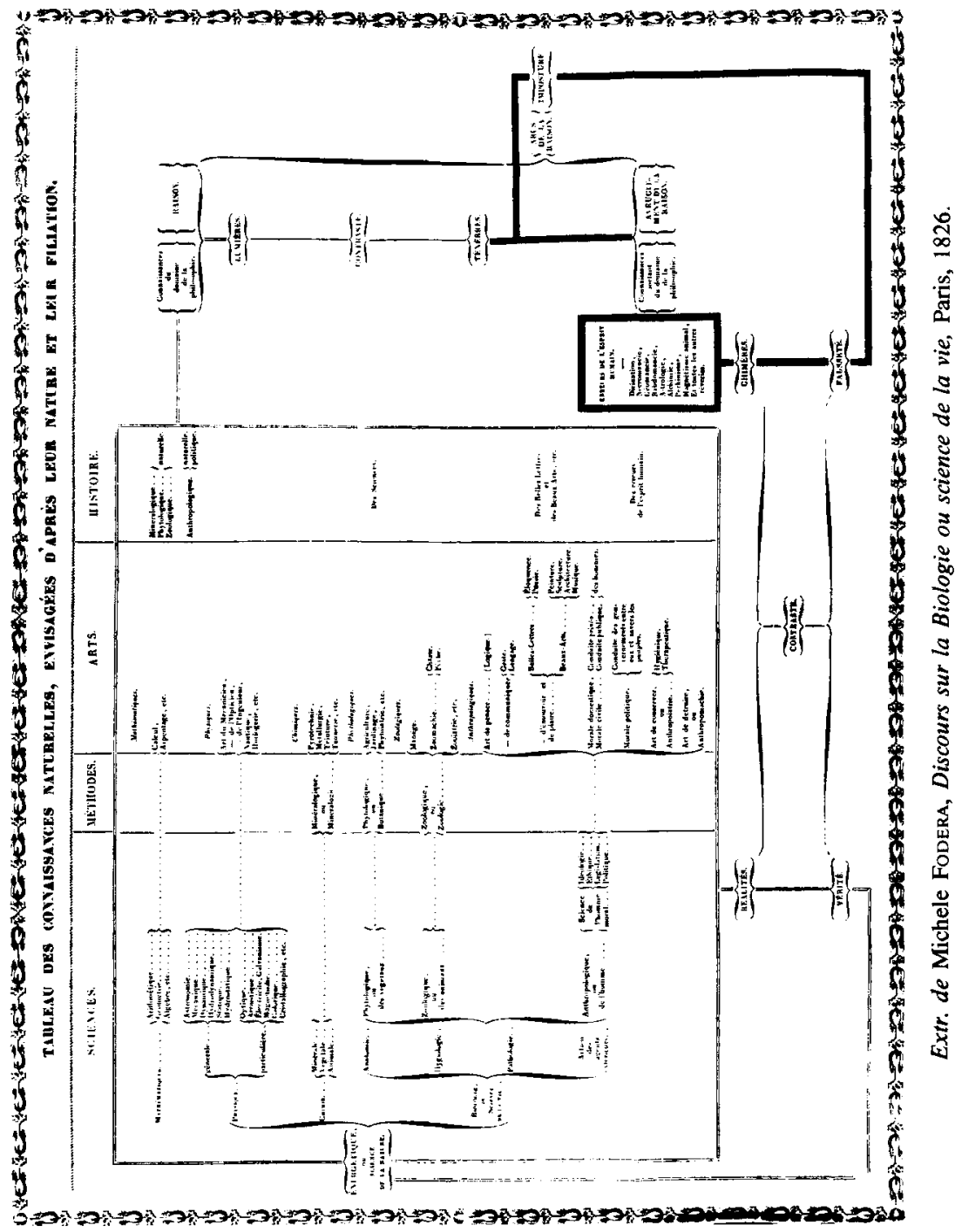


entrée "physiologie ou science de l'organisation ", dans un article qui distingue une biologie animale et une biologie végétale; Blainville, dans l'introduction de son Cours de physiologie comparée (1829), l'utilise dans la définition de la zoobiologie ou zoobie, partie de la biologie, et c'est en 1845, qu'il fait son entrée dans le Dictionnaire de médecine de LittréRobin, comme partie de la physiologie, en même temps que la nouvelle définition de la cellule. Par-delà l'évolution des termes et ce jeu d'éclipses entre 1800 et l'installation définitive du terme " biologie ", par-delà la vie et l'histoire des mots, auxquels l'identité d'une discipline scientifique est liée, se pose la question des conditions complexes qui permettent de définir une science et son institutionnalisation et, dans le cas de la biologie, le problème du critère de pertinence de sa définition : nature de l'objet, méthode d'étude, niveau de validité de l'unification du monde vivant, rapport avec les autres disciplines, etc. Peut-être cette construction historique peut-elle rappeler que, même dans des domaines où l'intuition première d'une différence radicale du vivant parait s'imposer naturellement, c'est bien un long et patient travail d'élaboration théorique de l'objet " biologie " qui a été produit historiquement; cette élaboration ne s'est pas faite seulement grâce à l'avènement de la méthode expérimentale, ou grâce au développement de la théorie cellulaire, ou à l'introduction d'un point de vue physiologique dans les sciences naturelles, et la problématique transformiste y a joué sa partie. Elle a aussi pris appui sur des philosophies, des conceptions de la nature ou de la vie qui sont devenues, aux yeux de la rationalité scientifique moderne, illégitimes. À l'heure où la biochimie occupe une place croissante - et l'existence même de ce terme hybride souligne la précarité des clivages entre disciplines scientifiques -, à l'heure où la biologie est devenue moléculaire, peut-être cette étude historique peut-elle rappeler que rien ne garantit $a$ priori la pérennité de « la biologie " comme catégorie définitive de la classification des sciences.

Roselyne REY, C.N.R.S.-Centre Alexandre-Koyré, Muséum national d'histoire naturelle, Pavillon Chevreul, 57, rue Cuvier, 75231 Paris Cedex 05 (1991). 